\title{
On Generalized Pell and Pell-Lucas Numbers
}

\author{
Lucyna Trojnar-Spelina ${ }^{1}$ (D) I Iwona Włoch ${ }^{1}$
}

Received: 20 February 2019/Accepted: 9 August 2019/Published online: 28 August 2019

(C) The Author(s) 2019

\section{Abstract}

In this paper, we introduce and study a new one-parameter generalization of Pell numbers. We describe their distinct properties also related to matrix representation.

Keywords Fibonacci numbers · Pell numbers $\cdot$ Lucas numbers $\cdot$ Pell-Lucas numbers $\cdot k$-Fibonacci numbers $k$-Pell numbers

Mathematics Subject Classification 11B37 $11 \mathrm{C} 20 \cdot 15 \mathrm{~B} 36 \cdot 05 \mathrm{C} 69$

\section{Introduction}

Let $F_{n}$ be the $n$th Fibonacci number defined recursively by $F_{n}=F_{n-1}+F_{n-2}$, for $n \geq 2$ with $F_{0}=F_{1}=1$. There are the well-known numbers of the Fibonacci type defined by the linear recurrence relation. We list some of them.

Lucas numbers:

$$
\begin{aligned}
& L_{n}=L_{n-1}+L_{n-2}, \\
& \quad \text { for } \quad n \geq 2 \quad \text { with } L_{0}=2, L_{1}=1,
\end{aligned}
$$

Pell numbers:

$$
\begin{aligned}
& P_{n}=2 P_{n-1}+P_{n-2}, \\
& \quad \text { for } \quad n \geq 2 \quad \text { with } P_{0}=0, P_{1}=1,
\end{aligned}
$$

Pell-Lucas numbers:

$$
\begin{aligned}
& Q_{n}=2 Q_{n-1}+Q_{n-2}, \\
& \quad \text { for } \quad n \geq 2 \quad \text { with } Q_{0}=Q_{1}=1,
\end{aligned}
$$

Let $k \geq 1$ be an integer. Then, we have $k$-Fibonacci numbers (Falcon and Plaza 2007):

Lucyna Trojnar-Spelina

1spelina@prz.edu.pl

Iwona Włoch

iwloch@prz.edu.pl

1 Department of Discrete Mathematics, Faculty of Mathematics and Applied Physics, Rzeszów University of Technology, Powstańców Warszawy Avenue 12, 35-959 Rzeszow, Poland

$$
\begin{aligned}
& F_{k, n}=k F_{k, n-1}+F_{k, n-2}, \\
& \quad \text { for } n \geq 2 \quad \text { with } F_{k, 0}=0, F_{k, 1}=1,
\end{aligned}
$$

and $k$-Pell numbers (Catarino 2013):

$$
\begin{aligned}
& P_{k, n}=2 P_{k, n-1}+k P_{k, n-2}, \\
& \quad \text { for } n \geq 2 \quad \text { with } P_{k, 0}=0, P_{k, 1}=1 .
\end{aligned}
$$

In this paper, we introduce and study a new one-parameter generalization of the Pell numbers. Let $k \geq 2, n \geq 0$ be integers. We define generalized Pell numbers $P_{k, n}$ recurrently as follows:

$$
\begin{aligned}
& P_{k, n}=k P_{k, n-1}+(k-1) P_{k, n-2} \\
& \quad \text { for } n \geq 2,
\end{aligned}
$$

with initial conditions $P_{k, 0}=0$ and $P_{k, 1}=1$. Next, we define generalized Pell-Lucas numbers $Q_{k, n}$ satisfying the recursive recurrence of the form:

$$
\begin{aligned}
& Q_{k, n}=k Q_{k, n-1}+(k-1) Q_{k, n-2} \\
& \quad \text { for } n \geq 2,
\end{aligned}
$$

with initial conditions $Q_{k, 0}=Q_{k, 1}=2$.

The tables presented below contain initial terms of the sequences $\left\{P_{k, n}\right\}$ and $\left\{Q_{k, n}\right\}$ for selected values of $k$ (Tables 1 and 2).

As we can see, for $k=2$ the classical Pell numbers and classical Pell-Lucas numbers are obtained. Sequences $\left\{P_{4, n}\right\},\left\{P_{5, n}\right\},\left\{P_{6, n}\right\},\left\{P_{7, n}\right\}$ are listed in The Online Encyclopaedia of Integer Sequences (OEIS Foundation Inc. 2018) under the symbols A015530, A015537, A0155551 and A015564, respectively, while sequences 
Table 1 Initial terms of the generalized Pell numbers $P_{k, n}$

\begin{tabular}{llllrrrrrrrr}
\hline$n$ & 0 & 1 & 2 & \multicolumn{1}{c}{3} & \multicolumn{1}{c}{4} & 5 & 6 & 7 & 8 & 9 & 10 \\
\hline$P_{2, n}$ & 0 & 1 & 2 & 5 & 12 & 29 & 70 & 169 & 408 & 985 & 2378 \\
$P_{3, n}$ & 0 & 1 & 3 & 11 & 39 & 139 & 495 & 1763 & 6279 & 22,363 & 79,647 \\
$P_{4, n}$ & 0 & 1 & 4 & 19 & 88 & 409 & 1900 & 8827 & 41,008 & 190,513 & 885,076 \\
$P_{5, n}$ & 0 & 1 & 5 & 29 & 165 & 941 & 5365 & 30,589 & 174,405 & 994,381 & $5,669,525$ \\
$P_{6, n}$ & 0 & 1 & 6 & 41 & 276 & 1861 & 12,546 & 84,581 & 570,216 & $3,844,201$ & $25,916,286$ \\
$P_{7, n}$ & 0 & 1 & 7 & 55 & 427 & 3319 & 25,795 & 200,479 & $1,558,123$ & $12,109,735$ & $94,116,883$ \\
\hline
\end{tabular}

Table 2 Initial terms of the generalized Pell-Lucas numbers $Q_{k, n}$

\begin{tabular}{lrrrrrrrrrrrr}
\hline$n$ & 0 & 1 & 2 & 3 & 4 & 5 & 6 & 7 & 8 & 9 & \multicolumn{1}{c}{10} \\
\hline$Q_{2, n}$ & 2 & 2 & 6 & 14 & 34 & 82 & 198 & 478 & 1154 & 2786 & 6726 \\
$Q_{3, n}$ & 2 & 2 & 10 & 34 & 122 & 434 & 1546 & 5506 & 19,610 & 69,842 & 248,746 \\
$Q_{4, n}$ & 2 & 2 & 14 & 62 & 290 & 1346 & 6254 & 29,054 & 134,978 & 627,074 & $2,913,230$ \\
$Q_{5, n}$ & 2 & 2 & 18 & 98 & 562 & 3202 & 18,258 & 104,098 & 593,522 & $3,384,002$ & $17,313,532$ \\
$Q_{6, n}$ & 2 & 2 & 22 & 142 & 962 & 6482 & 43,702 & 294,622 & $1,986,242$ & $12,212,079$ & $75,258,716$ \\
$Q_{7, n}$ & 2 & 2 & 26 & 194 & 1514 & 11,762 & 91,418 & 710,498 & $5,521,994$ & $42,916,946$ & $333,550,586$ \\
\hline
\end{tabular}

$\left\{\frac{1}{2} Q_{2, n}\right\},\left\{\frac{1}{2} Q_{4, n}\right\},\left\{\frac{1}{2} Q_{5, n}\right\},\left\{\frac{1}{2} Q_{6, n}\right\}$, under the symbols A001333, A086901, A123270 and A123362, respectively.

The recurrence relations (1.6) and (1.7) generate characteristic equation of the form

$r^{2}-k r+1-k=0$.

Since $k \geq 2$, this equation has two roots

$r_{1}=\frac{1}{2}\left(k-\sqrt{k^{2}+4 k-4}\right)$,

$r_{2}=\frac{1}{2}\left(k+\sqrt{k^{2}+4 k-4}\right)$.

Let us observe that for $k \geq 2$ we have $r_{1}<0<r_{2}<1$ and $\left|r_{1}\right|<r_{2}$. Moreover, the following interrelationships hold true:

$r_{1}+r_{2}=k$

$r_{1}-r_{2}=-\sqrt{k^{2}+4 k-4}$

$r_{1} r_{2}=1-k$

Next we find generating functions for the generalized Pell numbers $P_{k, n}$. Let us suppose that $P_{k, n}$ are coefficients of a power series with center at the origin and that $f_{k}(x)$ is the sum of this series, i.e.,

$f_{k}(x)=\sum_{n=0}^{\infty} P_{k, n} \cdot x^{n}$.

Such an analytic function $f_{k}$ is generating function for this sequence. Taking into account (1.6) and using the initial conditions $P_{k, 0}=0$ and $P_{k, 1}=1$, we get after some calculations

$$
\begin{aligned}
f_{k}(x) & =\sum_{n=0}^{\infty} P_{k, n} \cdot x^{n} \\
& =P_{k, 0}+P_{k, 1} \cdot x+\sum_{n=2}^{\infty}\left[k P_{k, n-1}+(k-1) P_{k, n-2}\right] x^{n} \\
& =x+k x \sum_{n=2}^{\infty} P_{k, n-1} x^{n-1}+(k-1) x^{2} \sum_{n=2}^{\infty} P_{k, n-2} x^{n-2} \\
& =x+k x \sum_{n=1}^{\infty} P_{k, n} x^{n}+(k-1) x^{2} \sum_{n=0}^{\infty} P_{k, n} x^{n} .
\end{aligned}
$$

Now by using (1.13), we obtain $f_{k}(x)=x+[k x+(k-$ 1) $\left.x^{2}\right] \cdot f_{k}(x)$, and then the generating function for the sequence $\left(P_{k, n}\right)$ is of the form

$f_{k}(x)=\frac{x}{1-k x-(k-1) x^{2}}$.

Now we find generating functions for the sequence $\left\{Q_{k, n}\right\}$. Let a generating function for this sequence be denoted by $g_{k}(x)$. Then, we have

$$
g_{k}(x)=\sum_{n=0}^{\infty} G_{k, n} \cdot x^{n} \text {. }
$$

Using recurrence (1.7) and initial conditions $Q_{k, 0}=Q_{k, 1}=$ 2 we have

$$
\begin{aligned}
g_{k}(x) & =\sum_{n=0}^{\infty} Q_{k, n} \cdot x^{n} \\
& =Q_{k, 0}+Q_{k, 1} \cdot x+\sum_{n=2}^{\infty}\left[k Q_{k, n-1}+(k-1) Q_{k, n-2}\right] x^{n} \\
& =2+2 x+k x \sum_{n=2}^{\infty} Q_{k, n-1} x^{n-1}+(k-1) x^{2} \sum_{n=2}^{\infty} Q_{k, n-2} x^{n-2} \\
& =2+2 x+k x \sum_{n=1}^{\infty} Q_{k, n} x^{n}+(k-1) x^{2} \sum_{n=0}^{\infty} Q_{k, n} x^{n} .
\end{aligned}
$$


After taking into account (1.15), we get $g_{k}(x)=2+2 x-$ $2 k x+\left[k x+(k-1) x^{2}\right] \cdot g_{k}(x)$ and therefore

$g_{k}(x)=\frac{2[1-(k-1) x]}{1-k x-(k-1) x^{2}}$.

\section{Explicit Formulas and Its Applications}

In this section, we give explicit formulas for the general terms of the sequences $\left\{P_{k, n}\right\}\left\{Q_{k, n}\right\}$ and next apply them to derive some identities. We start with the following theorem.

Theorem 2.1 The n-th terms of the sequences $\left\{P_{k, n}\right\}$ and $\left\{Q_{k, n}\right\}$ are of the form

$P_{k, n}=\frac{r_{1}^{n}-r_{2}^{n}}{r_{1}-r_{2}}$

$Q_{k, n}=\frac{2\left(1-r_{2}\right)}{r_{1}-r_{2}} r_{1}^{n}+\frac{2\left(r_{1}-1\right)}{r_{1}-r_{2}} r_{2}^{n}$,
Now we give the identity showing the relationship between numbers $P_{k, n}$ and $Q_{k, n}$.

Corollary 2.2 For all integers $k \geq 2$ and $n \geq 0$ we have

$Q_{k, n}=2\left[P_{k, n}+(k-1) P_{k, n-1}\right]$.

Proof We will make some transformations of the $Q_{k, n}$ given by the formula (2.2)

$$
\begin{aligned}
Q_{k, n} & =\frac{2}{r_{1}-r_{2}}\left[r_{1}^{n}-r_{1}^{n} r_{2}+r_{1} r_{2}^{n}-r_{2}^{n}\right] \\
& =\frac{2}{r_{1}-r_{2}}\left[\left(r_{1}^{n}-r_{2}^{n}\right)-r_{1} r_{2}\left(r_{1}^{n-1}-r_{2}^{n-1}\right)\right] \\
& =2\left[\frac{r_{1}^{n}-r_{2}^{n}}{r_{1}-r_{2}}-r_{1} r_{2} \frac{r_{1}^{n-1}-r_{2}^{n-1}}{r_{1}-r_{2}}\right] .
\end{aligned}
$$

Now, after applying (1.12) and (2.1), we immediately obtain the required result (2.5).

As a consequence of Binet's formulas for the sequences $\left\{P_{k, n}\right\}$ and $\left\{Q_{k, n}\right\}$, the following results can be obtained:

Corollary 2.3 For all integers $k \geq 2$ and $n \geq 0$

$$
\begin{aligned}
P_{k, n} & =\frac{1}{2^{n} \sqrt{k^{2}+4 k-4}} \times\left[\left(k+\sqrt{k^{2}+4 k-4}\right)^{n}-\left(k-\sqrt{k^{2}+4 k-4}\right)^{n}\right] \\
Q_{k, n} & =\frac{\left(2-k+\sqrt{k^{2}+4 k-4}\right)\left(k+\sqrt{k^{2}+4 k-4}\right)^{n}-\left(2-k-\sqrt{k^{2}+4 k-4}\right)\left(k-\sqrt{k^{2}+4 k-4}\right)^{n}}{2^{n} \sqrt{k^{2}+4 k-4}} .
\end{aligned}
$$

respectively, where $r_{1}, r_{2}$ are given in (1.9).

Proof Since the characteristic Eq. (1.8) has two distinct roots $r_{1}, r_{2}$, the numbers $r_{1}^{n}, r_{2}^{n}$ are linearly invariant and form the basis for the space of all solutions of the Eqs. (1.6) and (1.7). Therefore

$P_{k, n}=a\left(r_{1}\right)^{n}+b\left(r_{2}\right)^{n}$

is the solution of the recurrence (1.6). Putting $P_{k, 0}=0$ and $P_{k, 1}=1$ we obtain $a+b=0$ and $a r_{1}+b r_{2}=1$. Solving this system of linear equations, we get $a=-b=\frac{1}{r_{1}-r_{2}}$. After putting these values of $a$ and $b$ in (2.3), we obtain (2.1) as required. We will use the analogical reasoning for the sequence $\left\{Q_{k, n}\right\}$. Then, the solution of the recurrence (1.7) is the following

$$
Q_{k, n}=c\left(r_{1}\right)^{n}+d\left(r_{2}\right)^{n} \text {. }
$$

Giving to $n$ the values $n=0$ and $n=1$ and solving the system of equations created in this way, we get the unique solution $(c, d)$ where $c=\frac{2\left(1-r_{2}\right)}{r_{1}-r_{2}}$ and $d=\frac{-2\left(1-r_{1}\right)}{r_{1}-r_{2}}$. Substituting these values in (2.4), we obtain (2.2).
In particular case, if $k=2$, we have the well-known formulas for the Pell sequence and the Pell-Lucas sequence, respectively.

$$
\begin{aligned}
& P_{n}=\frac{\sqrt{2}}{4}\left[(1+\sqrt{2})^{n}-(1-\sqrt{2})^{n}\right], \\
& Q_{n}=(1+\sqrt{2})^{n}+(1-\sqrt{2})^{n} .
\end{aligned}
$$

Lemma 2.4 For all integers $k \geq 2$ and $n \geq 0$ we have

$\lim _{n \rightarrow \infty} \frac{P_{k, n+1}}{P_{k, n}}=r_{2}$.

Proof By using (2.1) we have

$\lim _{n \rightarrow \infty} \frac{P_{k, n+1}}{P_{k, n}}=\lim _{n \rightarrow \infty} \frac{r_{2}^{n+1}-r_{1}^{n+1}}{r_{2}^{n}-r_{1}^{n}}$

The relation $\left|r_{1}\right|<\left|r_{2}\right|$ implies that $\lim _{n \rightarrow \infty}\left(\frac{r_{1}}{r_{2}}\right)^{n}=0$ and therefore $\lim _{n \rightarrow \infty} \frac{P_{k, n+1}}{P_{k, n}}=r_{2}$.

For $k=2$, it can be obtained that $\lim _{n \rightarrow \infty} \frac{P_{n+1}}{P_{n}}=1+\sqrt{2}$ for the classical Pell sequence. The number $1+\sqrt{2}$ is known as silver ratio. For the sequence $P_{3, n}$ is $\lim _{n \rightarrow \infty} \frac{P_{3, n+1}}{P_{3, n}}=\frac{1}{2}(3+\sqrt{17})$. 
By Lemma 2.4, we obtain that the radius of convergence of the series given in (1.13) is equal to $\frac{1}{r_{2}}>1$. Therefore, we get the following equality for all integers $k \geq 2$

$f_{k}(x)=\sum_{n=0}^{\infty} P_{k, n} \cdot x^{n} \quad$ for $\quad x \in\left(-\frac{1}{r_{2}}, \frac{1}{r_{2}}\right)$.

The generating function can now be used to give another form of the general term of the sequence $\left(P_{k, n}\right)$.

Theorem 2.5 For all integers $k \geq 2$ and $n \geq 1$ we have $P_{k, n}=\frac{f_{k}^{(n)}(0)}{n !}$,

where $f^{(n)}$ denotes as usual the $n$-th-order derivative of the function $f(x)$.

Proof The desired result is a simple consequence of the well-known relationship between coefficients of the Maclaurin series for the function and a value at the point 0 of its $n$th derivative.

The Binet's formulas (2.1) and (2.2) can be very useful to derive some identities for $\left\{P_{k, n}\right\}$ and $\left\{Q_{k, n}\right\}$. We give them in the following theorem.

Theorem 2.6 (Catalan's identity) For any positive integer $r$ we have

$$
\begin{aligned}
& P_{k, n-r} P_{k, n+r}-P_{k, n}^{2}=-(1-k)^{n-r} P_{k, r}^{2}, \\
& Q_{k, n-r} Q_{k, n+r}-Q_{k, n}^{2}=-8(1-k)^{n+1-r} P_{k, r}^{2} .
\end{aligned}
$$

Proof First we will prove (2.6). By using (2.1) and (1.12), we obtain

$$
\begin{aligned}
P_{k, n-r} P_{k, n+r}-P_{k, n}^{2} \\
=\frac{r_{2}^{n-r}-r_{1}^{n-r}}{r_{2}-r_{1}} \cdot \frac{r_{2}^{n+r}-r_{1}^{n+r}}{r_{2}-r_{1}}-\left(\frac{r_{2}^{n}-r_{1}^{n}}{r_{2}-r_{1}}\right)^{2} \\
=\frac{2\left(r_{1} r_{2}\right)^{n}-r_{2}^{n-r} r_{1}^{n+r}-r_{1}^{n-r} r_{2}^{n+r}}{\left(r_{2}-r_{1}\right)^{2}} \\
=\frac{\left(r_{1} r_{2}\right)^{n}}{\left(r_{2}-r_{1}\right)^{2}}\left[2-\left(\frac{r_{1}}{r_{2}}\right)^{r}-\left(\frac{r_{2}}{r_{1}}\right)^{r}\right] \\
=\frac{(1-k)^{n}}{\left(r_{2}-r_{1}\right)^{2}}\left[2-\frac{r_{1}^{2 r}+r_{2}^{2 r}}{\left(r_{1} r_{2}\right)^{r}}\right] \\
=\frac{-(1-k)^{n}}{(1-k)^{r}\left(r_{2}-r_{1}\right)^{2}} \times\left[r_{1}^{2 r}-2\left(r_{1} r_{2}\right)^{r}+r_{2}^{2 r}\right] \\
=-(1-k)^{n-r}\left(\frac{r_{2}^{r}-r_{1}^{r}}{r_{2}-r_{1}}\right)^{2} .
\end{aligned}
$$

Repeated application of (2.1) gives the required result. In the proof of (2.7), we use the Binet's formula (2.2) for $Q_{k, n}$. Putting $c=\frac{2\left(1-r_{2}\right)}{r_{1}-r_{2}}$ and $d=\frac{2\left(r_{1}-1\right)}{r_{1}-r_{2}}$, we have

$$
\begin{aligned}
Q_{k, n-r} & Q_{k, n+r}-Q_{k, n}^{2} \\
= & \left(c r_{1}^{n-r}+d r_{2}^{n-r}\right)\left(c r_{1}^{n+r}+d r_{2}^{n+r}\right)-\left(c r_{1}^{n}+d r_{2}^{n}\right)^{2} \\
= & c^{2} r_{1}^{2 n}+c d\left(r_{1}^{n-r} r_{2}^{n+r}+r_{1}^{n+r} r_{2}^{n-r}\right) \\
& +d^{2} r_{2}^{2 n}-c^{2} r_{1}^{2 n}-2 c d r_{1}^{n} r_{2}^{n}-d^{2} r_{2}^{2 n} \\
= & c d\left(r_{1} r_{2}\right)^{n}\left[\left(\frac{r_{1}}{r_{2}}\right)^{r}+\left(\frac{r_{2}}{r_{1}}\right)^{r}-2\right] \\
= & -4\left(1-r_{1}\right)\left(1-r_{2}\right) \frac{\left(r_{1} r_{2}\right)^{n}}{\left(r_{1}-r_{2}\right)^{2}} \\
& \cdot \frac{\left(r_{1}\right)^{2 r}-2\left(r_{1} r_{2}\right)^{r}+\left(r_{2}\right)^{2 r}}{\left(r_{1} r_{2}\right)^{r}} \\
= & -4\left[1-\left(r_{1}+r_{2}\right)+r_{1} r_{2}\right]\left(r_{1} r_{2}\right)^{n-r} \times\left(\frac{r_{1}^{r}-r_{2}^{r}}{r_{1}-r_{2}}\right)^{2} .
\end{aligned}
$$

Now using (1.10) and (1.12) we obtain $Q_{k, n-r} Q_{k, n+r}-$ $Q_{k, n}^{2}=-8(1-k)^{n+1-r} P_{k, r}^{2}$ as required.

For $k=2$, the following identities can be deduced:

$P_{n-r} P_{n+r}-P_{n}^{2}=(-1)^{n+1-r} P_{r}^{2}$,

$Q_{n-r} Q_{n+r}-Q_{n}^{2}=8(-1)^{n+2-r} P_{r}^{2}$.

First of them is the well-known Catalan's identity for Pell numbers. Giving to $r$ the value 1, we can write two identities $P_{n-1} P_{n+1}-P_{n}^{2}=(-1)^{n} \quad$ and $\quad Q_{n-1} Q_{n+1}-Q_{n}^{2}=$ $8(-1)^{n+1}$ known as Simpson formulas for the Pell numbers (Horadam 1971) and for the Pell-Lucas numbers (Horadam and Mahon 1985).

Now we give next identities in the following theorem.

Theorem 2.7 Let $m, n$ be positive integers and $m \geq n$. Then, we have

$P_{k, m} P_{k, n+1}-P_{k, m+1} P_{k, n}=(1-k)^{n} P_{k, m-n}$

and

$Q_{k, m} Q_{k, n+1}-Q_{k, m+1} Q_{k, n}=8(1-k)^{n+1} P_{k, m-n}$.

Proof Similarly that before we use (2.1). After some calculation, we obtain

$$
\begin{aligned}
P_{k, m} P_{k, n+1}-P_{k, m+1} P_{k, n} \\
=\frac{-1}{\left(r_{2}-r_{1}\right)^{2}}\left[r_{1}^{n+1} r_{2}^{m}+r_{1}^{m} r_{2}^{n+1}-r_{1}^{n} r_{2}^{m+1}-r_{1}^{m+1} r_{2}^{n}\right] \\
=\frac{1}{\left(r_{2}-r_{1}\right)^{2}}\left[r_{1}^{n} r_{2}^{m}\left(r_{2}-r_{1}\right)-r_{1}^{m} r_{2}^{n}\left(r_{2}-r_{1}\right)\right] \\
=\left(r_{1} r_{2}\right)^{n} \frac{r_{2}^{m-n}-r_{1}^{m-n}}{r_{2}-r_{1}} .
\end{aligned}
$$

Then, the conclusion can be directly obtained by (1.12) and (2.1).

Note that for $k=2$ d'Ocagne's identity for Pell numbers appears: 


$$
P_{m} P_{n+1}-P_{m+1} P_{n}=(-1)^{n} P_{m-n} .
$$

Here, next identities for generalized Pell numbers and generalized Pell-Lucas numbers will be shown. They will allow to express the sum of the first terms of the sequences $\left\{P_{k, n}\right\}$ and $\left\{Q_{k, n}\right\}$ in concise form.

Theorem 2.8 For all integers $k \geq 2, n \geq 0$ we have

$$
\sum_{i=0}^{n} P_{k, i}=\frac{1}{2(k-1)} \times\left[(k-1) P_{k, n}+P_{k, n+1}-1\right]
$$

and

$$
\sum_{i=0}^{n} Q_{k, i}=\frac{1}{2(k-1)}\left[(k-1) Q_{k, n}+Q_{k, n+1}+2 k-4\right] .
$$

Proof Note that

$\sum_{i=0}^{n} P_{k, i}=\frac{1}{r_{2}-r_{1}}\left[\sum_{i=0}^{n} r_{2}^{n}-\sum_{i=0}^{n} r_{1}^{n}\right]$.

Thus, by summing up the geometric partial sums, we obtain

$$
\begin{aligned}
& \sum_{i=0}^{n} P_{k, i}=\frac{1}{r_{2}-r_{1}}\left(\frac{1-r_{2}^{n+1}}{1-r_{2}}-\frac{1-r_{1}^{n+1}}{1-r_{1}}\right) \\
& \quad=\frac{\left(1-r_{2}\right)^{n+1}\left(1-r_{1}\right)-\left(1-r_{1}\right)^{n+1}\left(1-r_{2}\right)}{\left(r_{2}-r_{1}\right)\left(1-r_{1}\right)\left(1-r_{2}\right)} \\
& \quad=\frac{\left(r_{2}-r_{1}\right)-\left(r_{2}^{n+1}-r_{1}^{n+1}\right)+r_{1} r_{2}\left(r_{2}^{n}-r_{1}^{n}\right)}{\left(r_{2}-r_{1}\right)\left(1-r_{1}\right)\left(1-r_{2}\right)} \\
& \quad=\frac{1}{\left(1-r_{1}\right)\left(1-r_{2}\right)} \times\left[1-\frac{r_{2}^{n+1}-r_{1}^{n+1}}{r_{2}-r_{1}}+r_{1} r_{2} \frac{r_{2}^{n}-r_{1}^{n}}{r_{2}-r_{1}}\right] .
\end{aligned}
$$

Now, again by using (1.12) and (2.1) and by noting that $\left(1-r_{1}\right)\left(1-r_{2}\right)=2(1-k)$, the result is obtained. For the proof of the identity (2.11) we use Binet's formula (2.2) for $Q_{k, n}$ with $c=\frac{2\left(1-r_{2}\right)}{r_{1}-r_{2}}$ and $d=\frac{2\left(r_{1}-1\right)}{r_{1}-r_{2}}$. Then, we obtain

$$
\begin{aligned}
\sum_{i=0}^{n} Q_{k, i} & =\sum_{i=0}^{n}\left(c r_{1}^{n}+d r_{2}^{n}\right)=c \sum_{i=0}^{n} r_{1}^{n}+d \sum_{i=0}^{n} r_{2}^{n} \\
& =c \frac{1-r_{2}^{n+1}}{1-r_{2}}+d \frac{1-r_{1}^{n+1}}{1-r_{1}} \\
& =\frac{c\left(1-r_{1}^{n+1}\right)\left(1-r_{2}\right)+d\left(1-r_{2}^{n+1}\right)\left(1-r_{1}\right)}{\left(1-r_{1}\right)\left(1-r_{2}\right)} \\
& =\frac{(c+d)-\left(c r_{2}+d r_{1}\right)-\left(c r_{1}^{n+1}+d r_{2}^{n+1}\right)+r_{1} r_{2}\left(c r_{1}^{n}+d r_{2}^{n}\right)}{\left(1-r_{1}\right)\left(1-r_{2}\right)} .
\end{aligned}
$$

A simple calculation gives $c+d=2$ and $c r_{2}+d r_{1}=$ $2(k-1)$. Therefore, after using (1.12) and once more the equality $\left(1-r_{1}\right)\left(1-r_{2}\right)=2(1-k)$ and Binet's formula for $Q_{k, n}$ we get

$$
\begin{aligned}
\sum_{i=0}^{n} Q_{k, i} & =\frac{1}{1-k}+2-\frac{1}{2(1-k)} Q_{k, n+1}+\frac{1}{2} Q_{k, n} \\
& =\frac{1}{2(k-1)}\left[(k-1) Q_{k, n}+Q_{k, n+1}+2 k-4\right] .
\end{aligned}
$$

The proof is completed.

As a consequence of (2.10) and (2.11), we can give the following identities for Pell numbers and Pell-Lucas numbers:

$$
\begin{aligned}
\sum_{i=0}^{n} P_{i} & =\frac{1}{2}\left(P_{n}+P_{n+1}-1\right) \\
\sum_{i=0}^{n} Q_{i} & =\frac{1}{2}\left(Q_{n}+Q_{n+1}\right) .
\end{aligned}
$$

\section{Application of Matrices}

One of the most popular methods for study of the sequences defined recursively is to use matrices. Application of the so-called matrix generator or generating matrix not only allows us to derive many identities for given sequence but also provides relatively simple proofs of them. It is well known that the numbers of the Fibonacci sequence are generated by so-called $Q$-matrix

$$
Q=\left[\begin{array}{ll}
1 & 1 \\
1 & 0
\end{array}\right]
$$

since by taking successive positive powers of $Q$ one can obtain

$$
Q^{n}=\left[\begin{array}{cc}
F_{n+1} & F_{n} \\
F_{n} & F_{n-1}
\end{array}\right] \text {. }
$$

The authorship of the name $Q$-matrix is assigned to King (1960) by Gould (1981) which in his article included more historical details about the $Q$-matrix, its other versions and its generalizations. In 1979, Silvester (1979) derived the properties of the Fibonacci numbers from its matrix representation. Bicknell (1975) showed that

$$
M=\left[\begin{array}{ll}
2 & 1 \\
1 & 0
\end{array}\right]
$$

is the generating matrix for the Pell sequence. A constructive method of finding all possible matrix generators of Pell sequence was given by Ercolano (1979) who showed that all these matrices are related to each other. Also in recent decades many authors use the matrix generators method to study a variety of sequences defined by recursion. For example, in Bednarz et al. (2013) and Włoch et al. (2013) the authors construct matrix representations of Fibonacci numbers generalized in the distance sense, while 
in Catarino (2013), Catarino and Vasco (2013) two-by-two matrices are applied to derive Cassini identities for $k$-Pell and $k$-Lucas sequences. In another recent paper Spivey (2006), the sum property of determinants is used to give a new proof of the well-known identity for Fibonacci sequence $F_{m} F_{n}-F_{m-r} F_{n+r}=(-1)^{m-r} F_{r} F_{n+r-m}$. The next example of using matrix generators can be found in the paper Dasdemir (2011).

The permanent of a matrix $A=\left[a_{i, j}\right]_{n \times n}$ is defined as follows:

$\operatorname{per} A=\sum_{\sigma \in S_{n}} a_{1, \sigma(1)} a_{2, \sigma(2)} \ldots a_{n, \sigma(n)}$

where $S_{n}$ denotes the symmetric group of all permutations of the indices $\{1,2, \ldots, n\}$, see, e.g., Minc (1978). The permanent of a square matrix is very similar to its determinant. The difference is that, in the case of permanent, all the signs occurring in the Laplace expansion of minors, are positive. The matrix $A=\left[a_{i, j}\right]_{m \times n}$ with row vectors $r_{1}, r_{2}, \ldots, r_{m}$ and column vectors $c_{1}, c_{2}, \ldots, c_{n}$ is called contractible on column $k$ if its column $c_{k}$ has exactly two nonzero elements. Similarly, $A$ is called contractible on row $k$ provided its row $r_{k}$ has exactly two nonzero elements. Let $A$ be contractible on column $k$ with two different nonzero entries $a_{i, k}$ and $a_{j, k}$ where $i \neq j$. Let us transform matrix $A$ by replacing its $i$ th row $r_{i}$ with $a_{j, k} r_{i}+a_{i, k} r_{j}$ and then deleting row $r_{j}$ and column $c_{k}$. This new $(m-1) \times$ $(n-1)$ matrix is called contraction of $A$ on column $k$ relative to rows $i$ and $j$ (Brualdi 2006).

We shall use contractions to prove that numbers $P_{k, n}$ can be expressed as permanents of some matrix. For integer $n \geq 1$, let $D_{n}(k)=\left[d_{i, j}\right]_{n \times n}$ denote tridiagonal matrix whose entries on the main diagonal are equal to $k$, entries on the subdiagonal are 1 , on the super-diagonal equal to $k-1$ and the other entries equal 0 . In other words, nonzero entries of this matrix are the following $d_{i, i}=k, d_{i, i+1}=k-1$, $d_{i+1, i}=1, i=1,2, \ldots, n$. This definition gives

$D_{n}(k)=\left[\begin{array}{cccccc}k & k-1 & 0 & \ldots & 0 & 0 \\ 1 & k & k-1 & \ldots & 0 & 0 \\ 0 & 1 & k & \ldots & 0 & 0 \\ \ldots & \ldots & \ldots & \ldots & \ldots & \ldots \\ 0 & 0 & 0 & \ldots & 1 & k\end{array}\right]$.

Let us recall the useful result

Lemma 3.1 (Brualdi 2006) Let $A$ be a nonnegative integral matrix of order $n>1$ and $B$ be a contraction of $A$. Then perA $=$ perB.

Then, we have the following theorem.

Theorem 3.2 Let $n \geq 1, \quad k \geq 2$ be integers. Then $\operatorname{perD}_{n}(k)=P_{k, n+1}$.
Proof For $n=1$ we have $\operatorname{per} D_{1}(k)=\operatorname{per}[k]=k P_{k, 1}+$ $(k-1) P_{k, 0}=P_{k, 2}$. For $n=2$ we obtain

$D_{2}(k)=\left[\begin{array}{cc}k & k-1 \\ 1 & k\end{array}\right]$

and $\operatorname{perD}_{2}(k)=k^{2}+k-1=k P_{k, 2}+(k-1) P_{k, 1}=P_{k, 3}$. All contractions of the matrices that will appear in what follows will be contractions on the column 1 relative to rows 1 and 2 . Let $D_{n}^{(i)}$ denote the matrix being $i$ th such contraction of the matrix $D_{n}(k)$. Of course, $D_{n}^{(i)}$ has dimensions $(n-i) \times(n-i)$. Let $n \geq 2$. Then, first contraction of the matrix $D_{n}(k)$ has a form

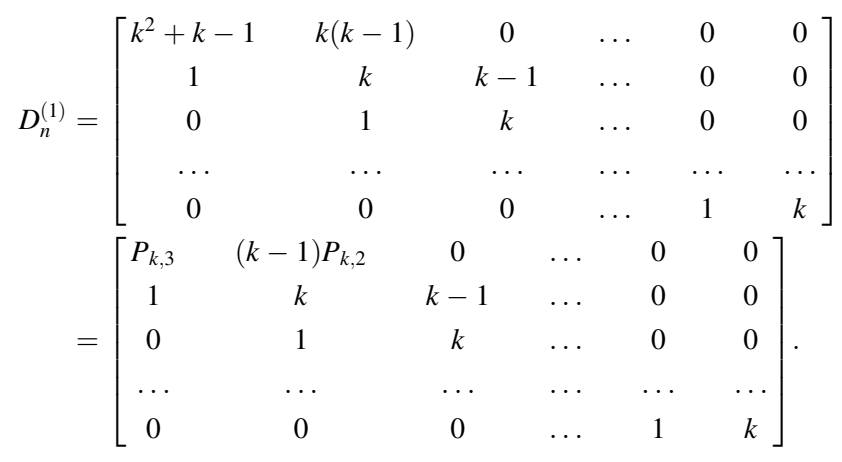

Contraction of $D_{n}^{(1)}$ becomes

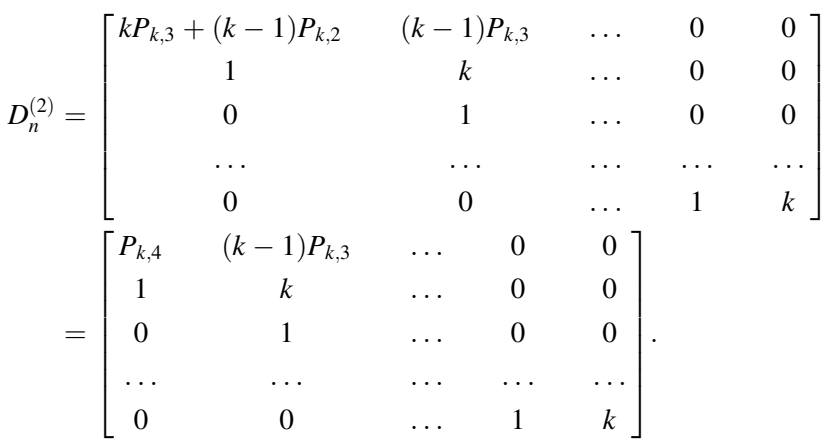

In general, for $1 \leq s \leq n-4$, we obtain

$$
D_{n}^{(s)}=\left[\begin{array}{ccccc}
P_{k, s+2} & (k-1) P_{k, s+1} & \ldots & 0 & 0 \\
1 & k & \ldots & 0 & 0 \\
0 & 1 & \ldots & 0 & 0 \\
\ldots & \ldots & \ldots & \ldots & \ldots \\
0 & 0 & \ldots & 1 & k
\end{array}\right] .
$$

Therefore, $D_{n}^{(n-3)}$ is the following $3 \times 3$ matrix

$D_{n}^{(n-3)}=\left[\begin{array}{ccc}P_{k, n-1} & (k-1) P_{k, n-2} & 0 \\ 1 & k & k-1 \\ 0 & 1 & k\end{array}\right]$

and after the last contraction appears 


$$
\begin{aligned}
D_{n}^{(n-2)} & =\left[\begin{array}{cc}
k P_{k, n-1}+(k-1) P_{k, n-2} & (k-1) P_{k, n-2} \\
1 & k
\end{array}\right] \\
& =\left[\begin{array}{cc}
P_{k, n} & (k-1) P_{k, n-1} \\
1 & k
\end{array}\right] .
\end{aligned}
$$

Hence, by Lemma 3.1 and by definition of generalized Pell numbers, we get

$$
\begin{aligned}
\operatorname{perD}_{n}(k) & =\operatorname{perD}_{n}^{(n-2)} \\
& =k P_{k, n}+(k-1) P_{k, n-1}=P_{k, n+1} .
\end{aligned}
$$

Open Access This article is distributed under the terms of the Creative Commons Attribution 4.0 International License (http://creative commons.org/licenses/by/4.0/), which permits unrestricted use, distribution, and reproduction in any medium, provided you give appropriate credit to the original author(s) and the source, provide a link to the Creative Commons license, and indicate if changes were made.

\section{References}

Bednarz U, Włoch A, Wołowiec-Musiał M (2013) Distance Fibonacci numbers, their interpretations and matrix generators. Comment Math 53(1):35-46

Bicknell N (1975) A primer on the Pell sequence and related sequence. Fibonacci Quart 13(4):345-349
Brualdi RH (2006) Combinatorial matrix classes. Encyclopaedia of mathematics and its applications, vol 108. Cambridge University Press, Cambridge

Catarino P (2013) A note involving two-by-two matrices of the k-Pell and k-Pell-Lucas sequences. Int Math Forum 8(32):1561-1568

Catarino P, Vasco P (2013) Some basic properties and a two-by-two matrix involving the k-Pell numbers. Int $\mathrm{J}$ Math Anal 7(45):2209-2215

Dasdemir A (2011) On the Pell, Pell-Lucas and modified Pell numbers by matrix method. Appl Math Sci 5(64):3173-3181

Ercolano J (1979) Matrix generators of Pell sequence. Fibonacci Quart 17(1):71-77

Falcon S, Plaza A (2007) On the Fibonacci $k$-numbers. Chaos Solitions Fractals 32(5):1615-1624

Gould AW (1981) A history of the Fibonacci Q-matrix and a higherdimensional problem. Fibonacci Quart 19(3):250-257

Horadam AF (1971) Pell identities. Fibonacci Quart 9(3):245-263

Horadam AF, Mahon JM (1985) Pell and Pell-Lucas polynomials. Fibonacci Quart 23(1):7-20

King CH (1960) Some properties of the Fibonacci numbers. Master Thesis, San Jose State College

Minc H (1978) Permanents Encyclopaedia of Mathematics and its Applications, vol 6. Addison-Wesley Publishing Company, London

OEIS Foundation Inc. (2018) The on-line encyclopedia of integer sequences. http://oeis.org

Silvester JR (1979) Fibonacci properties by matrix methods. Math Gaz 63:188-191

Spivey MZ (2006) Fibonacci identities via determinant sum property. Coll Math J 37:286-289

Włoch I, Bednarz U, Bród D, Włoch A, Wołowiec-Musiał M (2013) On a new type of distance Fibonacci numbers. Dis Appl Math 161:2695-2701 